\title{
MicroRNAs keep microglia quiet
}

MicroRNAs (miRNAs) regulate gene expression in many biological processes, and a new study shows that a brain-specific miRNA is a key regulator of microglial cell quiescence in the CNS, thereby helping to prevent CNS inflammation.

Microglial cells are CNS-resident macrophages that, under normal conditions, have a resting phenotype that is characterized by low-level expression of CD45 and major histocompatibility complex (MHC) class II molecules. During CNS inflammation, such as that which occurs in experimental autoimmune encephalomyelitis (EAE), microglial cells become activated and are thought to contribute - together with peripheral macrophages that infiltrate the inflamed CNS - to the pathological

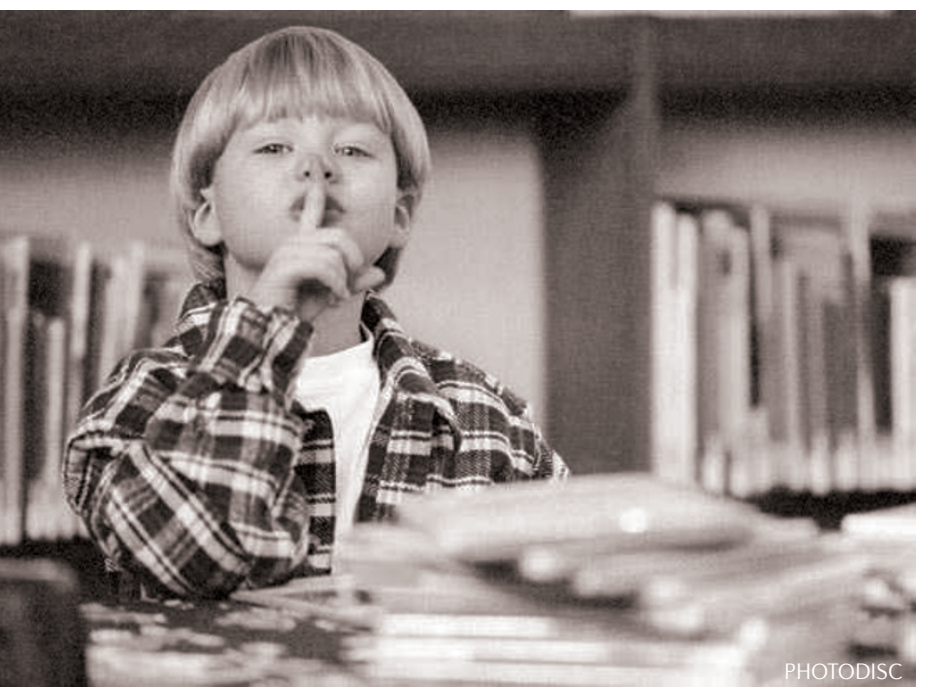

processes. The mechanisms that maintain the unique resting phenotype of microglial cells are unknown, so Weiner and colleagues set out to investigate whether miRNAs might be involved.

A comparison of expression of 31 known miRNAs in macrophages isolated from different organs of healthy adult mice revealed that only CNS-resident microglial cells expressed the miRNA miR-124. During EAE, however, miR-124 expression by microglial cells decreased, and CNS-infiltrating peripheral macrophages started to express low levels of miR-124 during the onset and recovery phases of the disease. This suggested that miR-124 expression correlates inversely with the activation state of microglial cells and macrophages in the CNS.

Consistent with the idea that miR-124 regulates the activation state of macrophages, the authors showed that transfection of bone marrowderived macrophages with miR-124 results in downregulation of activation markers, including CD45, MHC class II and CD86. miR-124 transfection also inhibited macrophage proliferation, promoted macrophage polarization to an M2 phenotype and altered cell morphology. Investigation of the mechanisms underlying these effects showed that miR-124 directly binds to the mRNA that encode the myeloid cell master transcription factor CCAAT/enhancer-binding protein- $\alpha$
(C/EBPa), causing downregulation of $\mathrm{C} / \mathrm{EBP} a$ protein expression.

Accordingly, mutation of the three predicted miR-124 binding sites in C/EBPa mRNA abolished the inhibitory effect. In turn, the reduced levels of C/EBPa in miR-124-transfected cells caused decreased expression of the downstream transcription factor PU.1 and its target genes encoding CD45 and MHC class II.

Returning to the in vivo setting, the authors showed that administration of miR-124 shortly after induction of EAE substantially ameliorated or prevented disease symptoms. Mice treated with miR-124 had less microglial cell activation and leukocyte infiltration in the CNS than control mice. These effects on disease were dependent on miR-124-mediated downregulation of $\mathrm{C} / \mathrm{EBP} \alpha$, as they could be replicated by a small interfering RNA specific for C/EBPa but not by miR-124 lacking the $\mathrm{C} / \mathrm{EBPa}$ binding sequence.

Finally, evidence from co-culture experiments support the hypothesis that signals from CNS stromal cells, such as astrocytes and neurons, cause macrophages to adopt a resting microglial cell phenotype through upregulation of miR-124.

Lucy Bird, Chief Editor,

Nature Reviews Immunology

ORIGINAL RESEARCH PAPER Ponomarev, E. D.

et al. MicroRNA-124 promotes microglia quiescence and suppresses EAE by deactivating macrophages via the C/EBP- $\alpha$-PU.1 pathway. Nature Med. 17, 64-70 (2011) 